\title{
La calle como campo. Caracterización de la situación de calle en Ibagué $e^{1}$
}

The street' as a field. Characterization concerning the streel' plight in lbagué

\author{
Juan David Zabala Sandoval²
}

(2).......

Recepción: 08/08/2020
(-)

Aprobación: 25/09/2020
-

Publicación: 18/12/2020

\section{Para citar este artículo:}

Zabala Sandoval, J. D. (2020). La calle como campo. Caracterización de la situación de calle en Ibagué. Indagare, (8), 58-68. https://doi.org/10.35707/indagare/806

\section{(c) $(i)(8)$}

\footnotetext{
${ }^{1}$ Este estudio implicó la formación del Semillero Desde el Margen, grupo con el que se llevó a cabo la formulación del proyecto, el trabajo de campo, la sistematización y divulgación de la información. Este proyecto se realizó en el marco de acción del grupo de investigación GESS, Universidad de Ibagué, Colombia.

${ }^{2}$ Estudiante del Doctorado en Ciencias Sociales, Universidad de Chile. ORCID: 0000-0001-8999-4053. Correo electrónico: juan.zabala@unibague.edu.co,juand.zabalas@gmail.com
} 


\section{Resumen}

La presente investigación explora y describe la situación de calle a partir del caso de Ibagué, dado que enfatiza en fenómenos característicos que permiten la estructuración de un habitus de calle, a partir de la idea de que la calle misma es un campo. Se empleó una metodología de corte cualitativo, diseño transeccional, con enfoque históricohermenéutico que se centró en la comuna uno del municipio. Se logró una caracterización básica de elementos estructurales, así como la identificación de trayectorias de vida, rutas y rutinas de desplazamiento, formas de arraigo e identidades y prácticas de explotación y de violencia sobre el cuerpo de manera diferencial entre sexos y fenómenos psicológicos, tales como desesperanza aprendida y derelicción.

\section{Abstract}

This research explores and describes the street plight based on the case of Ibagué, since it stresses in characteristic phenomena that allow the structuring of a street habitus, from the idea that the street itself is a field. A qualitative methodology, a cross-sectional design, with a historical-hermeneutical approach that focused on Commune One of the municipality were used. A basic characterization of structural elements was achieved, as well as the identification of life paths, routes and commuting routines, forms of rooting and identities, and practices of exploitation and violence against the body in a differential way between sexes and psychological phenomena, such as learned hopelessness and dereliction.

\footnotetext{
Palabras claves: Situación de calle, identidad, marginalidad, exclusión social.

Key words: Street plight, identity, marginality, social exclusion.
}

\section{Introducción}

La situación de calle representa una condición característica de nuestras sociedades contemporáneas altamente gentrificadas en urbes extensas, basadas en una lógica de competencia e individualismo, apoyadas en la separación o distanciamiento emocional entre los individuos y, paradójicamente, en el aumento de su interdependencia (Simmel, 2014). El hecho de vivir en la calle es un fenómeno que no es exclusivo, pero sí representativo de las ciudades, a la vez que es un signo de la desigualdad y de la vulnerabilidad de la vida en las sociedades contemporáneas (Correa, 2007). 
La habitanza de calle es una condición compleja y multidimensional que se relaciona con problemáticas como la exclusión social, la pobreza, la precariedad y el consumo de sustancias psicoactivas (SPA), que condicionan las posibilidades de llevar una vida digna y que se establecen como constantes en las ciudades occidentales (Germani, 1973; Ministerio de Protección Social, 2006). Por esto, debe pensarse como un producto del sistema social, económico y de las dinámicas mismas de interacción, que cotidianamente lo actualizan. De esta manera, se evita pensarlo como una suma de accidentes aislados o el resultado de la inadecuación de los individuos ante las exigencias del medio. La presente investigación es exploratoria y descriptiva y busca fundamentar planteamientos de mayor precisión y profundidad a nivel teórico y aplicado, de manera tal que el aporte es también de mayor provecho para la ciudadanía.

Para el Ministerio de Protección Social de Colombia (2006), existe una diferenciación entre el habitante de calle y el habitante en calle, que alude a que el segundo tiene una morada fija o por lo menos dispone de un lugar en donde pasar la noche. Ambos tienen por característica la falta de ingresos propios, no cuentan con un empleo o poseen un trabajo en condiciones precarias; que se traduce en serias dificultades para cubrir sus necesidades. Los dos encuentran en la calle los medios para satisfacer sus necesidades, además, hacen de este lugar no solo su medio de subsistencia sino también su hogar (Berho, 2006; 2010; Ley 1641 de 2013).

En la dimensión social y ciudadana se evidencia la falta de equidad en cuanto a la posibilidad de atención, servicios y recursos que permitan suplir las necesidades de esta población (Gómez Urueta, 2013; Quintero, 2008). Esto se traduce en limitaciones frente al acceso a una identificación, a servicios de salud, educación, entre otros. Lo cual configura una constelación de factores relativos a las condiciones de precariedad y pobreza extrema.

En adición, estas personas se encuentran fragmentadas o han sido invisibilizadas frente a agentes administrativos, por tanto, se ven obligadas a habitar en la calle como si fuera su aposento, razón primaria para el alto grado de vulnerabilidad al que están expuestos, pues además de la inclemencia del clima, también son objeto de violencias físicas, simbólicas, económicas y sexuales, por mencionar algunas (Palleres, 2010). Pero en especial, se denota la poca existencia, casi nula, de redes de apoyo y relaciones duraderas e íntimas, al punto de hablar de ruptura con la familia (Berho, 2006; Cortés, 2006); estos son los elementos que configuran el fenómeno básico de la habitanza en calle (De Souza, 2015; Moreno, 2001; Quintero, 2008). 
En resumen, es habitante de calle quien además de pasar la mayor parte de su tiempo diario, vive o pasa la noche a la intemperie, resultado de la inestabilidad en contar con un lugar fijo, adecuado y digno de residencia, o simplemente de no contar con alguno, por lo cual su participación social es mínima, hecho que lo define como un marginal (Bachiller, 2010; Cortés, 2006).

\subsection{La calle como campo}

Se podría definir la habitanza en calle como una condición social e históricamente producida que lleva a situaciones de desventaja. Esta circunstancia está marcada por limitaciones en la participación efectiva en sociedad, relaciones de desigualdad y violencia, así como la dificultad para llevar una vida digna; debido a que propone escenarios alternos en los cuales es posible otros órdenes no necesariamente supeditados a las lógicas de distribución de capitales de la sociedad hegemónica. En consecuencia, podría plantearse que la calle habría de constituir un campo en sí misma, con sus reglas y prácticas propias, en el sentido que plantea Bourdieu (2008).

Así, vivir en la calle articula una serie de elementos estructuralmente dispuestos que tienden a reproducirse a sí mismos, a la vez que dificultan que un sujeto pueda salir de ella. De esta manera, lo ejemplifica Del Monte Madrigal (2018), con su concepto del vórtice de precarización, puesto que propone la articulación de diferentes condiciones, como el consumo de SPA, la ruptura de vínculos afectivos, la carencia económica, entre otros. Estas situaciones marcan, de a poco, la entrada de las personas en la calle como la acumulación de incertidumbres y la ausencia de apoyo institucional/estatal, que llega a deteriorar la agencia misma del individuo y hace más difícil el proceso de salida que el de entrada a la vida en la calle.

Sin embargo, no se debe recaer en el planteamiento errado del individualismo metodológico, que supone que las formas particulares de pensar o actuar de los individuos explican las condiciones estructurales, como la pobreza, la marginalidad, entre otras expresiones de la precariedad. Antes bien, se espera estudiar ¿cómo las situaciones materiales, históricas y socialmente producidas tienen una reverberación a nivel de los individuos de modo que implican una reproducción de dicha condición? Es así que la habitanza en calle podría llevar a una serie de aprendizajes, esquemas de comportamiento o desviaciones en las concepciones que los individuos hacen del mundo y de sí mismos, que facilitan la reproducción de la desigualdad y el funcionamiento precario en el sistema, pero que, en contraposición, resultan funcionales dentro del campo de la calle. 
Bourdieu (2008) entendería que los diferentes intercambios que se presentan entre individuos y grupos reflejan la serie de reglas que dan forma al campo, visto como un entramado de relaciones que se estructura a partir de principios o pilares que implican, en cierta manera, la ponderación diferencial de una serie de recursos (capitales). Cada campo permite a los actores tratar de posicionarse a partir del uso estratégico que se haga de los capitales de los cuales disponga, esto es sistemático. Por lo cual redunda en la aparición de prácticas, o mejor, conductas instrumentales iterativas que son legadas por los individuos a partir de su posicionamiento en el campo y su pertenencia en diversos grupos. Es así como el accionar del individuo se puede explicar a partir de la articulación de prácticas en habitus, que son disposiciones que se marcan en el cuerpo y que operan de forma independiente de la conciencia e intención del actor, al punto que aseguran la reproducción del orden establecido del campo mismo (Martuccelli \& Santiago, 2017).

Se hace necesario entablar esfuerzos de estudiar las formas en que los elementos estructurales y relacionales de la habitanza en calle, como la marginalidad se produce y reproduce a nivel microsocial, en tanto son encarnadas en las vidas de los individuos al punto incluso de ser asumidas, justificadas y validadas por estos. Por un lado, está la tarea de revisar la articulación entre lo estructural y las trayectorias de vida a partir de los desafíos sociales (Martuccelli, 2007), que configuran el proceso de individuación y subjetivación, de manera que se dé cuenta de las estrategias y prácticas que asumen los individuos con tal de sobrellevar la precariedad (Arteaga \& Pérez, 2011; Martuccelli \& Santiago, 2017), esto es, delimitar el habitus marginal y sus efectos en los procesos de individuación.

A la vez, es de interés mostrar las construcciones de identidad individual y social que emergen de estos estilos de vida. También sus reflejos simbólicos a nivel espacio temporal, o mejor, evidenciar esos procesos que ocurren con la gente que vive en la calle en términos de identidades, arraigos y relaciones (Berho, 2006). Esto, con tal de analizar la situación de calle en sus elementos estructurales, sus prácticas y dinámicas de reproducción evidenciadas en las disposiciones apropiadas por los actores sociales.

Se busca recolectar la información necesaria para dar respuesta a diversas preguntas con las cuales se pueda agrupar el concepto de identidad callejera y las implicaciones que su apropiación como estilo de vida posee en cuanto a exclusión, discriminación y marginalidad social. Lo anterior, al tener en cuenta la vulnerabilidad y la probabilidad de transgresión de derechos humanos, que se presume se encuentran expuestos en su día a día. Se contempla la posibilidad de aportar información que pudiese ayudar a pensar la política pública del municipio referente a esta problemática, así como a orientar planes de acción e intervención contextuados y, por lo tanto, más efectivos. 


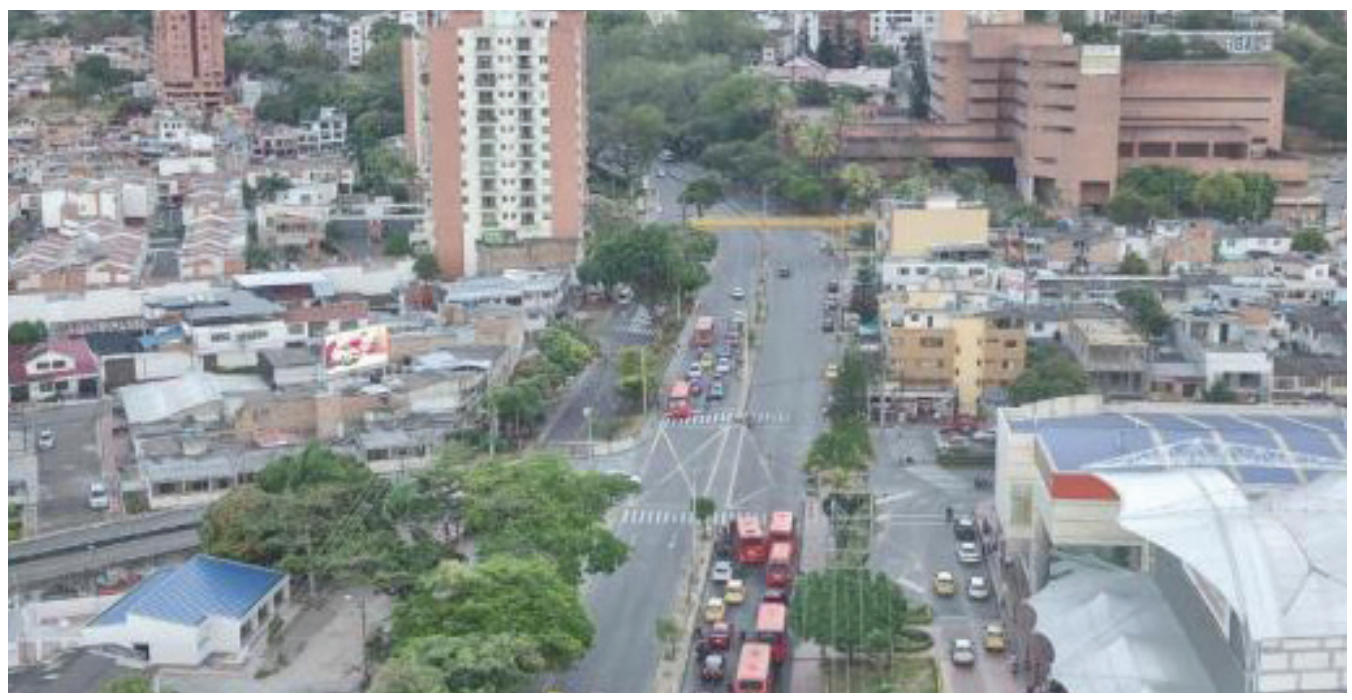

\section{Materiales y métodos}

El estudio se llevó a cabo con una metodología de corte cualitativo, diseño transeccional, con enfoque histórico-hermenéutico. Se trabajó con ciudadanos habilitantes de calle mayores de edad que generalmente se concentran en la comuna uno (1) de la ciudad de Ibagué. Se empleó una muestra por conveniencia, la metodología utilizada ocupó un enfoque construccionista e interaccionista con tres fases de aplicación: 1) aproximación y exploración de las calles de Ibagué - centro, comuna uno- a partir de entrevistas a informantes clave y observaciones; 2) descripción de la calle a partir de una aproximación multimetódica compuesta por entrevistas semiestructuradas (Ortiz, 2007), observación participante, diarios de campo y relatos de vida (AMOVI, 2016; Güelman \& Borda, 2014; Martínez Miguélez, 2006); 3) identificación de los conjuntos de prácticas y rutinas propias de la vida en la calle, a partir de talleres participativos de cartografías (Soliz \& Maldonado, 2012; Vélez, Rátiva \& Varela, 2012;) y etnografía. Este trabajo se llevó a cabo entre junio de 2017 y mayo de 2018.

\section{Resultados}

En datos del último censo, se denota un total de 624 habitantes de calle, 553 hombres y 71 mujeres. Se tienen datos detallados de 435 de ellos, la mayoría se encuentra entre las edades de 26-45 años (50\%), más de la mitad proviene del departamento del Tolima (55\%). En cuanto a sus condiciones, el $22 \%$ no tiene documento de identidad (cédula de ciudadanía), el $71 \%$ son solteros, el $49 \%$ no tiene acceso a servicios de salud, el $26 \%$ tiene alguna discapacidad física y el $6 \%$ alguna discapacidad mental. 
La mayoría, es decir, el $51 \%$ se ocupa en labores de reciclaje. En su plano educativo, el $13 \%$ dice no tener algún nivel de formación, el $48 \%$ haber cursado primaria y el $36 \%$ secundaria. Finalmente, en cuanto a las razones de ingreso a la calle, aparece el consumo de SPA con el $24 \%$, violencia intrafamiliar con el $23 \%$, dificultades económicas, con el $21 \%$ y desilusiones afectivas con el $18 \%$; mientras que en las razones de permanencia en la calle, el $29 \%$ refiere a dificultades económicas, el $22 \%$ a decisión personal, el $20 \%$ a falta de apoyo familiar y el $18 \%$ a consumo SPA (Alcaldía Municipal de Ibagué, 2018).

Resulta notorio el hecho de que al menos un $15 \%$ de la población encuestada afirma no consumir SPA, junto al hecho de que buena parte (41\%) dice haber entrado en esta situación por razones familiares o afectivas. Un sector relevante (22 \%) afirma su propia libertad a través de la decisión personal, así que, no es posible reducir la situación de calle al consumo de SPA, ni a la pobreza (económica ni multidimensional), ni a la exclusión social; antes bien, parece una combinación de condiciones que se acentúan con elementos propios de la acción, como las prácticas, e internos, como la sensación de fragilidad (López, 2019).

De manera general, la información obtenida apunta a una descripción de las formas en que las vivencias articulan trayectorias de vida reconocibles que llevan a las personas a vivir en la calle, tales como los conflictos y el maltrato intrafamiliar, problemáticas económicas, exclusión y violencia social, entre otras (Buriticá, 2018). Lo anterior es coincidente con aquello que apunta Del Monte Madrigal (2018) en su estudio; no obstante, el paso por estas vicisitudes puede conllevar la construcción de un individuo que toma de responsabilidad sobre sí mismo o, por el contrario, un aparente abandono que suele coincidir con lo comprendido como derelicción y desesperanzan aprendida (López, 2019). Esto es la resignación, la automarginación, la justificación de la situación de desigualdad vivida a partir de la dependencia y culpabilización de otros, el merecimiento dado a atributos inherentes y aprendidos del individuo y la predestinación (López, 2019; Méndez \& Ortiz, 2018).

Por otra parte, fue posible identificar que la vida en las calles de Ibagué se focaliza alrededor de los centros económicos y sociales del municipio. En este caso constituyen territorios comunes a la vida en calle como parques, terminales, hogares de paso y avenidas comerciales, en los cuales transcurre la cotidianidad de estas personas (Bocanegra, 2018). Estos lugares son, a la vez, propicios para dinámicas de reconocimiento y menosprecio evidenciadas en prácticas puntuales que se convierten en hábitos y se describen en términos espaciales y temporales; por lo tanto, su estudio permite direccionar esfuerzos de futuras intervenciones. 
Los hogares de paso aparecen como instituciones clave que articulan la oferta de servicios y bienes de la sociedad mayoritaria para con la población en calle; se establecen como umbrales o fronteras de marginalidad, entre el centro de la sociedad mayoritaria y el centro de la sociedad de la calle. Por eso quienes asisten a estas instituciones quedan relegados de ambas y deben iniciar un tránsito hacia alguna de las dos. Por parte de los usuarios, los hogares de paso son vistos como facilitadores de bienes y servicios; en adición, se denota una estimación social y una valía inherente al hogar de paso, en forma de valores, reglas y estatus adquirido por pertenecer al mismo. Esto se traduce en un arraigo funcional y moral que se refuerza por las acciones de sociabilidad que allí tienen ocurrencia (Palacino Hartmann \& Gutiérrez, 2018).

Es así que los hogares de paso se establecen como centros de sociabilidad en las periferias sociales, razón por la cual se pudo evidenciar la constitución de identidades ligadas a la habitanza en calle y a la recurrencia al hogar de paso, que se superponen como una gama que va de una polaridad a la otra sin ser totalmente excluyentes, antes bien, se intersectan, dado que las formas de vida (hábitos) adquiridos en el hogar de paso son permeados hasta cierto punto por la calle.

Es necesario resaltar que la calle implica una serie de reglas que estructuran y son estructuradas por las interacciones que allí se manifiestan, esto es, un habitus de calle que denota las formas en que los actores juegan en aras de sobrevivir. Esto involucra, como ya lo señaló Bourdieu (2008), que necesariamente hay una diferenciación e incluso una jerarquización al interior del campo, con base en las capacidades de uso y disposición de capitales. En el caso de los habitantes de calle, su principal capital parece ser el cuerpo, que implica formas de vivir, transitar, trabajar, aguantar/soportar y ocupar. Para el caso específico de las mujeres, el cuerpo se constituye como frontera última de las violencias de las que son objeto, cuya amenaza constante de violación (Segato, 2003) aumenta el grado de vulnerabilidad que ya tiene el vivir en la calle (Arboleda, Giraldo \& Padilla, 2019).

\section{Potencial uso}

Como conclusiones, es necesario continuar con investigaciones que develen las interrelaciones de los diferentes factores que componen el fenómeno de la habitanza de calle, en especial como una forma de vida derivada de la violencia social como marginación de las formas centrales y mayoritarias de vida social, cultural, económica y política. Esta marginación es de prioritaria atención en términos de alcanzar mayor justicia social, mejor gobernanza y en aras de una sociedad que cumpla con las exigencias de una vida democrática en el sentido establecido por la Política Pública Social para

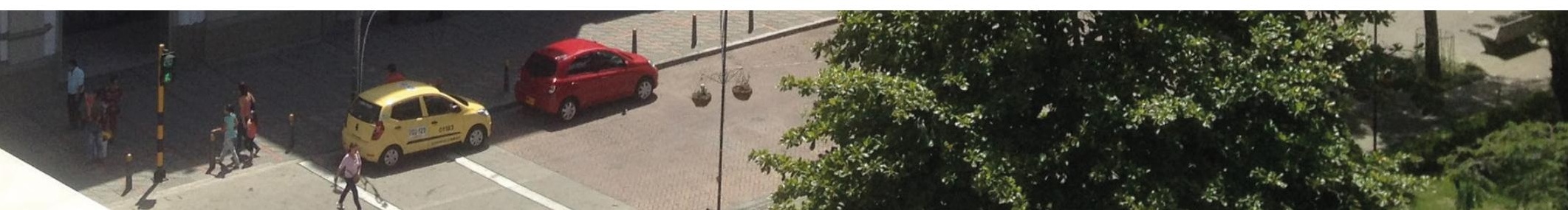


habitante de calle (MinSalud, 2018). De allí que se haya hecho una propuesta de ruta de atención psicosocial para habitantes de y en calle que se presentó ante la alcaldía municipal (Álvarez \& Meléndez, 2019).

También es necesario estudiar los grupos minoritarios de las minorías mismas, tales como las mujeres habitantes de calle y sus condiciones de vida, en tanto ellas encarnan dinámicas múltiples de exclusión. También es menester estudiar las poblaciones iniciales como forma de prevenir la habitanza en calle y tratar de reducir la reproducción de estas formas de vida; es así como el estudio de los niños en situación de calle aparece en el panorama.

Finalmente, con respecto a la población estudiada, debe plantearse formas de intervención acordes con los principios de igualdad, dignidad y autonomía. Por ello debe descartarse el enfoque asistencialista y propender por la participación activa y la extensión de esfuerzos en generalizar el conocimiento y el acceso a derechos en términos de capacidades, bienes y servicios como forma de hacer de la vida en la calle algo digno, de brindar posibilidades creíbles de salir de esta (si así se desea) y de prevenir que se llegue a esta situación. En ese sentido, se proponen abordajes interdisciplinarios que reconocen el potencial que tiene la educación popular y el arte, como la propuesta elaborada por el Semillero Desde el Margen de un protocolo de intervención basado en la metodología de las Colmenas de arte (Arteta \& Guerrero, 2019).

\section{Ficha técnica del proyecto}

Título del proyecto: Identidad, reconocimiento y marginalidad. Caracterización de la situación de calle en Ibagué.

PRIT: Desarrollo regional inclusivo y sustentable.

Código del proyecto: $19-497-$ INT.

Palabras claves: Situación de calle, identidad, marginalidad, exclusión social.

Grupo de investigación: Educación, Salud y Sociedad, GESS.

Investigador principal: Juan David Zabala Sandoval.

Correo electrónico: juan.zabala@unibague.edu.co

Semillero: Desde el Margen.

\section{Referencias}

Alcaldía Municipal de Ibagué. (2018). Censo y caracterización de habitantes de la calle. Fundación Aguapanelos de la Calle. Convenio 1689 del 13 de octubre. Ibagué, Colombia.

Álvarez, V., \& Meléndez, V. (2019). Diseño de ruta de atención psicosocial para habitantes de/en calle RAPSIHC (Trabajo de grado del programa de Psicología). Universidad de Ibagué, Tolima, Colombia. 
INDAGA3E e-ISSN: 2357-5042 • Número 8 (2020) • Universidad de Ibagué • doi: https://doi.org/10.35707/indagare/806

AMOVI. (2016). Archivo oral de memoria de las víctimas. Recuperado de https://n9.cl/qolww

Arboleda, J., Giraldo, A., \& Padilla, J. (2019). Condición de calle y vulnerabilidad del cuerpo en mujeres de la ciudad de Ibagué, a través de sus narrativas (Trabajo de grado del programa de Psicología). Universidad de Ibagué, Tolima, Colombia.

Arteaga, C., \& Pérez, S. (2011). Experiencias de vulnerabilidad: de las estrategias a las tácticas subjetivas. Universum, 26(2), 67-81. http://dx.doi.org/10.4067/S0718-23762011000200004

Arteta, A., \& Guerrero, J. (2019). Colmenas de arte como intervención psicosocial para la promoción de espacios de interacción y reconocimiento entre habitantes de calle y la ciudadanía (Trabajo de grado del programa de Psicología). Universidad de Ibagué, Tolima, Colombia.

Bachiller, S. (2010). El aislamiento social como supuesto articulador de las teorías sobre la exclusión y el sinhogarismo: críticas y aportes etnográficos. CUHSO, 19(1), 9-21. http://dx.doi.org/10.7770/cuhsoV19N1-art305

Berho, M. (2006). Identidad marginal entre personas sin hogar de la ciudad de Temuco, Chile. CUHSO, 11(1), 39-55. http://dx.doi.org/10.7770/cuhso-V11N1-art243

Berho, M. (2010). Dos relatos, un análisis y un excurso sobre las identidades y la relación con la ciudad entre los "moradores de la calle" en Temuco, Chile. CUHSO, 19(1), 23-36. http://dx.doi.org/10.7770/ cuhso-V19N1-art306

Bocanegra, J. (2018). Reconocimiento y menosprecio de los habitantes de calle en la ciudad de Ibagué: centro-comuna uno (Trabajo de grado del programa de Psicología). Universidad de Ibagué, Tolima, Colombia.

Bourdieu, P. (2008). El sentido práctico. A. Dilon (Trad.). Buenos Aires, Argentina: Siglo XXI editores.

Buriticá, M. A. (2018). Identidad narrativa y desafío social. Una mirada desde la experiencia del habitante de calle (Trabajo de grado del programa de Psicología). Universidad de Ibagué, Tolima, Colombia.

Colombia. Ley 1641 de 2013. Por la cual se establecen los lineamientos para la formulación de la política pública social para habitantes de la calle y se dictan otras disposiciones.

Correa, M. (2007). Para una nueva comprensión de las características y la atención social a los habitantes de calle. Eleuthera, (1), 91-102. Recuperado de http://eleuthera.ucaldas.edu.co/downloads/Revista1_6.pdf

Cortés, F. (2006). Consideraciones sobre la marginación, la marginalidad, marginalidad económica y exclusión social. Papeles de Población, 12(47), 71-84. Recuperado de https://www.redalyc.org/ articulo.oa?id=11204704

Del Monte Madrigal, J. (2018). El vórtice de precarización. El proceso de indigencia en una ciudad fronteriza del norte de México (Tesis de doctorado en Ciencia Social con especialidad en Sociología, Centro de Estudios Sociológicos, Colegio de México, Ciudad de México, México). Recuperado de https://n9.cl/b7ba

De Souza, I. (2015). A construção social do morador de rua: o controle simbólico da identidade (Tesis de maestría en Ciencias Sociales, Universidad Federal de Juiz de Fora, Minas Gerais, Brasil). Recuperado de https://repositorio.uff.br/jspui/handle/uff/2199

Germani, G. (1973). El concepto de marginalidad. Significado, raíces históricas y cuestiones teóricas, con particular referencia a la marginalidad urbana. Buenos Aires, Argentina: Ediciones Nueva Visión.

Gómez Urueta, C. (2013). El habitante de calle en Colombia: presentación desde una perspectiva socialpreventiva. Recuperado de https://bit.ly/2Smuzl2

Güelman, M., \& Borda, P. (2014). Narrativas y reflexividad: los efectos biográficos del enfoque biográfico. Revista Latinoamericana de Metodología de las Ciencias Sociales, 4(1), 1-16. Recuperado de www.acuedi.org/ddata/7261.pdf 
INDAGA3E e-ISSN: 2357-5042 • Número 8 (2020) • Universidad de Ibagué • doi: https://doi.org/10.35707/indagare/806

López, M. (2019). Factores aprendidos de la permanencia de y en calle: desesperanza aprendida y pensamiento marginal (Trabajo de grado del programa de Psicología). Universidad de Ibagué, Tolima, Colombia. Martínez Miguélez, M. (2006). Ciencia y arte en la metodología cualitativa. México: Editorial Trillas.

Martuccelli, D. (2007). Cambio de rumbo. La sociedad a escala del individuo (1. ${ }^{a}$ ed.). Santiago de Chile, Chile: LOM ediciones.

Martuccelli, D., \& Santiago, J. (2017). El desafío sociológico hoy. Individuo y retos sociales. Madrid, España: Centro de Investigaciones Sociológicas (CIS).

Minsalud. (2018). Política Publica Social para habitante de calle -PPSHC-. Recuperado de https://n9.cl/4nvmh

Ministerio de Protección Social. (2006). Identificación, documentación y socialización de experiencias de trabajo con habitantes de y en calle (Informe final). Recuperado de https://n9.cl/2s3pi

Méndez, D., \& Ortiz, C. (2018). Relatos de vida y la marginalidad social de mujeres que ejercen prostitución (Trabajo de grado del programa de Psicología). Universidad de Ibagué, Tolima, Colombia.

Moreno, M. P. (2001). Psicología de la marginación social: concepto, ámbitos y actuaciones. Málaga, España: Ediciones Aljibe.

Ortiz, F. (2007). La entrevista de investigación en las ciencias sociales. México: Limusa Noriega Editores.

Palacino Hartmann, S., \& Gutiérrez, L. (2018). Arraigo y construcción de identidad en los habitantes de calle de la ciudad de Ibagué (Trabajo de grado del programa de Psicología, Universidad de Ibagué, Tolima, Colombia). Recuperado de https://hdl.handle.net/20.500.12313/1159

Palleres, G. (2010). Resignificación socioespacial y construcción de subjetividad. Personas sin hogar en la Ciudad de Buenos Aires. CUHSO, 19(1), 9-21. http://dx.doi.org/10.7770/cuhso-V19N1-art313

Quintero, L. (2008). La exclusión social de "habitantes de la calle" en Bogotá: una mirada desde la bioética. Revista Colombiana de Bioética, 3(1) 101-144. Recuperado de http://www.bioeticaunbosque.edu.co/ publicaciones/Revista/Revista5/Articulo4.pdf

Segato, R. L. (2003). Estructuras elementales de la violencia. Ensayos sobre género entre la antropología, el psicoanálisis y los derechos humanos (1. ${ }^{\mathrm{a}}$ ed.). Buenos Aires, Argentina: Universidad de Quilmes. Recuperado de http://repositorio.ciem.ucr.ac.cr/jspui/handle/123456789/156

Simmel, G. (2014). El pobre. Madrid, España: Ediciones Sequitur.

Soliz, F., \& Maldonado, A. (2012). Guía de metodologías comunitarias participativas. Guía n. ${ }^{\circ}$ 5. Recuperado de https://n9.cl/94t0

Vélez, I., Rátiva, S., \& Varela, D. (2012). Cartografía social como metodología participativa y colaborativa de investigación en el territorio afrodescendiente de la cuenca alta del río Cauca. Cuadernos de geografía: revista colombiana de geografía, 21(2), 59-73. Recuperado de https://revistas.unal.edu.co/ index.php/rcg/article/view/25774 